\title{
A high-temperature dielectric process as a probe of large-scale silica filler structure in simplified industrial nanocomposites
}

\author{
Guilhem P. Baeza ${ }^{1,2,3}$, Julian Oberdisse ${ }^{1,2}$, Angel Alegria ${ }^{4}$, Marc Couty $^{3}$ and Anne-Caroline \\ Genix $^{1,2^{*}}$ \\ ${ }^{1}$ Université Montpellier 2, Laboratoire Charles Coulomb UMR 5221, F-34095, Montpellier, \\ France \\ ${ }^{2}$ CNRS, Laboratoire Charles Coulomb UMR 5221, F-34095, Montpellier, France \\ ${ }^{3}$ Manufacture Française des Pneumatiques MICHELIN, Site de Ladoux, 23 place des \\ Carmes Déchaux, F-63040 Clermont-Ferrand, Cedex 9, France \\ ${ }^{4}$ Departamento de Física de Materiales y Centro de Física de Materiales CSIC-UPV/EHU, \\ Universidad del País Vasco UPV/EHU, Paseo Manuel de Lardizabal 5, E-20018 San
} Sebastian, Spain

\begin{abstract}
The existence of two independent filler-dependent high-temperature Maxwell-Wagner-Sillars dielectric processes (MWS) is demonstrated and characterized in detail in silica-filled styrenebutadiene (SB) industrial nanocomposites of simplified composition using Broadband Dielectric Spectroscopy (BDS). The uncrosslinked samples are made with $140 \mathrm{~kg} / \mathrm{mol} \mathrm{SB}$ chains, half of which carry a single graftable end-function (50\%D3), and Zeosil $1165 \mathrm{MP}$ silica incorporated by solid-phase mixing. While one high-temperature process is known to exist in other systems, the dielectric properties of a new silica-related process - strength, relaxation time, and activation energy - have been evidenced and described as a function of silica volume fraction and temperature. In particular, it is shown that its strength follows a percolation behavior as observed with the ionic conductivity and rheology. Moreover, activation energies show the role of polymer layers separating aggregates even when they are percolated. Apart from simultaneous characterization over a broad frequency range up to local polymer and silanol dynamics, it is believed that such high-temperature BDS-measurements can thus be used to detect reorganizations in structurally-complex silica nanocomposites. Moreover, they should contribute to a better identification of dynamical processes via the described sensitivity to structure in such systems.
\end{abstract}




\section{INTRODUCTION}

The structure of nanocomposites made of hard inorganic filler nanoparticles dispersed in a polymeric matrix determines many macroscopic properties (mechanical, optical, electrical, ... ${ }^{1-3}$ ) suitable for applications, like e.g. car tire optimization ${ }^{4}$. Dynamical properties are of equal importance for such applications, as it is easily recognized with rheological ones, the viscoelastic response of nanocomposites being strongly frequency-dependent. Indeed, modern car tires exploit this fact to decrease (low frequency) rolling resistance - and thus fuel consumption and $\mathrm{CO}_{2}$ production - without sacrificing (high frequency) wet grip. ${ }^{5}$ In general, nanocomposites have complex multi-scale structures, including the structure of polymer and of nanoparticles, which may arrange on different structural levels. The physical origin of the dynamical processes in such systems remains an open problem necessitating fundamental studies of both structure and dynamics. Broadband dielectric spectroscopy (BDS) offers insights into the dynamics of a variety of processes, from very high frequency and thus very local ones describing dynamics of individual functional groups, to polymer relaxations in presence of suitable dipolar moments, and finally to slow processes measurable only at the highest temperatures. The latter processes include the ionic conductivity, as well as MaxwellWagner-Sillars (MWS) processes.

MWS processes are related to polarization effects due to charge carrier diffusion through the different phases of nanocomposites. Such polarization results in the trapping and accumulation of charges at the polymer/silica interface, which is why they are structure dependent. As silica NPs are non-conducting, ions can diffuse on the surface of individual NPs within aggregates, or on a larger scale, either on the aggregate surface, or across polymer between aggregates. For slow, large-scale processes, the corresponding time scales are controlled both by the diffusion rate of charges and the characteristic distances to be covered, the latter being given by the multi-scale filler structure.

In a series of articles, the structure of simplified (i.e., without crosslinker and related chemistry) industrial silica-styrene-butadiene nanocomposites has been investigated as a function of silica content ${ }^{6}$, matrix composition in terms of graftable chains ${ }^{7}$, and chain mass ${ }^{8}$. The grafting density was identified as the key parameter by evidencing the existence of twin samples having a different composition but identical structure and elastic plateau moduli. ${ }^{9}$ By combining small-angle scattering, electron microscopy, and numerical simulations, we have described the structure of such complex systems by primary silica beads organized within 
small aggregates, which themselves fill large-scale fractal branches. The typical size and mass of the aggregates - some forty nanometers in radius, and aggregation numbers of about 45 beads - were deduced from TEM and SAXS data using a quantitative model of interacting aggregates involving an aggregate size polydispersity of $30 \%$. Such a high polydispersity translates into an even broader distribution in aggregate mass. The existence of several characteristic length scales (beads, aggregates, branches) indicates that dielectric processes may be supported by structures differing in size and nature and may thus occur on different time scales. In addition, a high polydispersity can translate in a broad distribution of scales for each process, to be captured with appropriate width parameters. In this article, we aim at correlating the evolution of the dynamical dielectric properties with the different scales of the microstructure of nanocomposite samples, with a special focus on large-scale structure. Such a correlation has been described in the literature for clay nanocomposites ${ }^{10}$. As far as we know, this is a novelty for dielectric spectroscopy of silica nanocomposites with multi-scale structure.

A first interfacial process (MWS1) has already been observed in several nanocomposite systems containing silica and carbon black. ${ }^{10-13}$ With carbon black, its characteristic time scale has been related to polymer layers between filler aggregates to be tunneled by charge carriers ${ }^{13}$, whereas an interlayer model based on hydration on the filler surface has been applied to silica nanocomposites ${ }^{11}$. It is the objective of the present article to describe a second interfacial process (MWS2), and show that it is affected in a very different manner by large-scale structural reorganizations as compared to MWS1. Moreover, the characteristic time of MWS2 will be shown to be unaffected by drying, whereas the one of MWS1 is shifted, which allows us to conclude on the contribution of water to the different processes. Such a MWS2 process has been partially identified for carbon black and related to reorganizations upon heat treatment and vulcanization ${ }^{13}$. Here it will be described in full detail for a silica-filled nanocomposite of well-described - albeit complex - microstructure.

\section{MATERIALS AND METHODS}

Materials. Details are given in ref ${ }^{6,7}$. The polymer matrix is made of styrene-butadiene (SB) random copolymers, with two types of chains of molecular mass $140 \mathrm{~kg} \cdot \mathrm{mol}^{-1}(\mathrm{PI}=1.07)$ as determined by size exclusion chromatography. Chains are either linear unmodified SB chains, or bear a single silanol end-function making the chains graftable onto the silica surface by 
condensation. These functionalized chains are denoted D3, and the matrix composition is fixed to $50 \%$ of D3 chains (denoted 50\%D3). Both types of chains have been purposesynthesized by Michelin. The silica pellets (Zeosil 1165 MP from Solvay) have a nominal specific surface of $160 \mathrm{~m}^{2} / \mathrm{g}$, and the size distribution of the nanoparticles obeys a log-normal law with $\mathrm{R}_{0}=8.55 \mathrm{~nm}, \sigma=27 \%$. ${ }^{6}$ Simplified industrial nanocomposites containing silica and SB are formulated by stepwise introduction and mixing of the chains with silica pellets in an internal mixer, keeping the final temperature constant at $160 \pm 5^{\circ} \mathrm{C}$. For simplicity, all the polymer additives, DPG (Vulcacit, Bayer, 1\%w with respect to polymer), the liquid coating agent octeo (Dynasylan, 8\% with respect to silica) and silica are incorporated simultaneously. Real silica volume fractions and residual water contents in all nanocomposites have been measured by thermogravimetry analysis (TGA), see ESI for details. The silica fractions lie in the range $\Phi_{\mathrm{si}}=8.4 \% \mathrm{v}-21.1 \% \mathrm{v}$ (i.e., between 21 and 63 phr), and water content is between 0.1 (matrix) and $1.8 \% \mathrm{w}$. The glass-transition temperature was determined using differential scanning calorimetry (Q2000 TMDSC, TA Instruments) in the modulated mode with a heating rate of $3 \mathrm{~K} / \mathrm{min}$. For the filled samples, $\mathrm{T}_{\mathrm{g}}$ shows no significant variation with silica content as compared to the pure polymer matrix: $T_{g}=-37 \pm$ $0.8^{\circ} \mathrm{C}$.

Broadband dielectric spectroscopy (BDS). Dielectric properties of the nanocomposites were measured on disk-shaped samples with a diameter of $30 \mathrm{~mm}$. All samples have been hot pressed $\left(60^{\circ} \mathrm{C}, 120\right.$ bars $)$ to reach a typical thickness of $0.15 \mathrm{~mm}$. Thin films were then placed between gold plate electrodes forming a capacitor. A small cross-shaped piece of Teflon (thickness $0.15 \mathrm{~mm}$ ) was included to prevent possible short-circuits due to polymer flow. A broadband high-resolution dielectric spectrometer (Novocontrol Alpha) was used to measure the complex dielectric permittivity $\varepsilon^{*}(\omega)=\varepsilon^{\prime}(\omega)$ - i $\varepsilon^{\prime}{ }^{\prime}(\omega)$ in the frequency range from $\mathrm{f}=10^{-2}$ to $10^{7} \mathrm{~Hz}(\omega=2 \pi \mathrm{f})$. After 20 minutes equilibration at $60^{\circ} \mathrm{C}$ in the BDS cryostat (under nitrogen atmosphere), isothermal frequency measurements have been performed with temperature stability better than $0.1 \mathrm{~K}$. To check the influence of water, samples have been measured up to $90^{\circ} \mathrm{C}$ first ("pre-drying"). Then, they have been dried in-situ at $100^{\circ} \mathrm{C}$ and $110^{\circ} \mathrm{C}$ for a total of one hour followed by an isotherm at $120^{\circ} \mathrm{C}$, where conductivity has been measured. A final set of measurements has been performed at lower temperatures again, now with a negligible water content ("post-drying", see ESI).

BDS data treatment. In order to get rid of possible artefacts coming from (i) different surface states of the samples (high roughness for high filler contents) and (ii) thickness 
variation due to temperature change, the complex permittivities have been normalized. This step consisted in applying a correction factor on both $\varepsilon^{\prime}(\omega, T)$ and $\varepsilon^{\prime \prime}(\omega, T)$ in order to have the high-frequency (low-temperature) limit of the real part of the permittivity, $\varepsilon_{\infty}$, equal to 2.5 . Such a value is directly related to the refractive index $\left(n \approx \varepsilon_{\infty}{ }^{0.5}\right)$. The chosen value is thus compatible with the refractive index of polymers, which is typically of the order of $1.3-1.7$ ${ }^{14}$. In our case, we have found $\mathrm{n}_{\mathrm{SB}}=1.53$, which is close to that of the silica filler refractive index according to the literature, $\mathrm{n}=1.54{ }^{15}$. This justifies taking a common normalization for different silica volume fractions. During the fitting procedure, we tried to systematically reduce the number of free parameters in the description of the dielectric spectra. This was done to extract reliable information in particular on time scales for strongly overlapping processes. A first fit of the data has been performed with all variables let free in the HavriliakNegami function given below, in order to extract possible trends with temperature for the shape parameters and the dielectric strength. Then, according to the results, these parameters were forced to follow a linear (possibly constant) behavior.

\section{RESULTS AND DISCUSSION}

Nanocomposites are made by solid-phase mixing of silica with SB-chains, half of which carry a single graftable end-function $(50 \% \mathrm{D} 3)^{6}$, and studied by BDS following the protocol given in section 2. We start with the lowest silica volume fraction, $8.4 \% \mathrm{vol}$, because it has the lowest ionic conductivity of the series, enabling a clearer view on the interfacial processes of interest. At high temperature $(\mathrm{T}=323 \mathrm{~K})$, slow dielectric relaxation processes designated MWS1 and MWS2 in Fig. 1 enter the frequency window. One can clearly see in the dielectric $\operatorname{loss} \varepsilon "(\omega)$ a low-frequency upturn characteristic of the ionic conductivity, and a strong and broad peak which cannot be described by a single process. In addition to the ionic dcconductivity, two distinct MWS-processes describing interfacial polarizations have to be considered to fit the data set correctly as illustrated in Fig. 1. Note here that the real part of the permittivity $\varepsilon^{\prime}(\omega)$ tends to reach a plateau at low frequencies supporting that the origin of this response is connected with the material dielectric relaxation and it is not simply due to electrode polarization effects ${ }^{16}$. Moreover, ionic mobility is linked to high-temperature polymer dynamics, and indeed $\mathrm{T}$ is greater than the glass-transition temperature $\mathrm{T}_{\mathrm{g}}$ of $236 \mathrm{~K}$ (see section 2). 


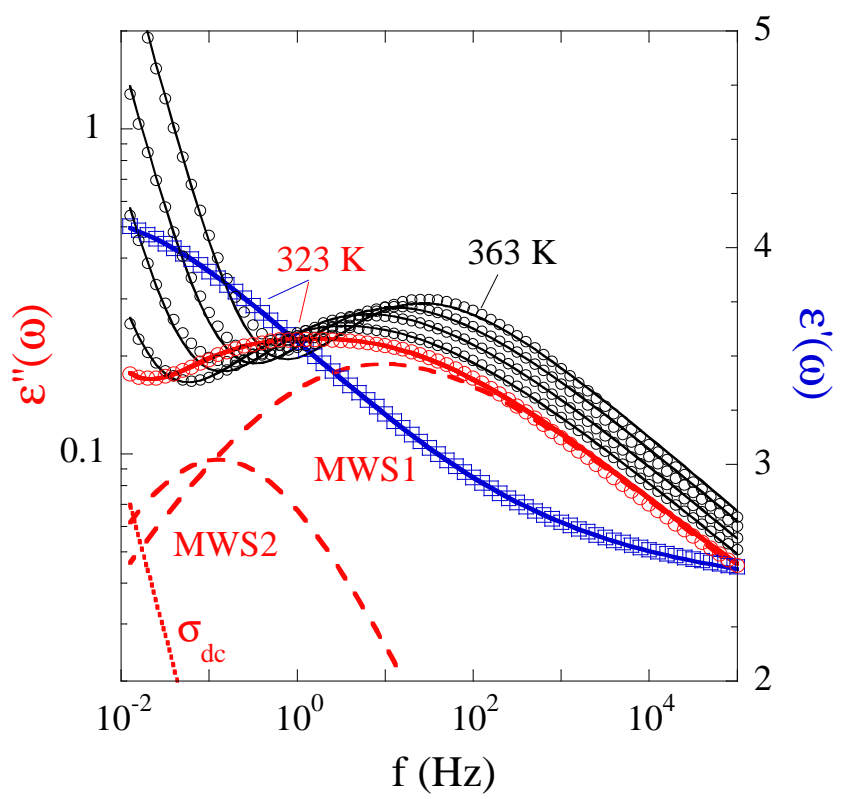

Fig. 1 Frequency dependence of the imaginary $(\varepsilon '$ ', circles) and real $(\varepsilon$ ', squares) parts of the complex dielectric permittivity measured on a nanocomposite with $8.4 \%$ vol silica $(50 \% \mathrm{D} 3)$ at $\mathrm{T}=323 \mathrm{~K}$. Additional data for $\varepsilon " '(\omega)$ at $\mathrm{T}=333$ up to $363 \mathrm{~K}$ each $10 \mathrm{~K}$ are shown in black. Solid lines through the data are fits according to eq 1 (fit parameters are given in the ESI). Dashed lines represent the individual contributions at $323 \mathrm{~K}$ as labeled in the figure.

A quantitative analysis of dielectric processes is usually performed in the frequency domain in terms of the empirical Havriliak-Negami (HN) function ${ }^{17}$. For each temperature, we combined the purely dissipative dc-conductivity term $\varepsilon{ }^{\prime}{ }_{\mathrm{dc}}=\sigma_{\mathrm{dc}} /\left(\varepsilon_{0} \omega\right)$ and one HN function for each MWS-relaxation present in the frequency window:

$$
\varepsilon^{*}(\omega)=\varepsilon_{\infty}+\sum_{j} \frac{\Delta \varepsilon_{j}}{\left[1+\left(i \omega \tau_{H N_{j}}\right)^{\gamma_{j}}\right]^{\delta_{j}}}-i \frac{\sigma_{d c}}{\varepsilon_{0} \omega}
$$

where $\gamma_{\mathrm{j}}$ and $\delta_{\mathrm{j}}$ are two shape parameters ranging between 0 and 1 describing the symmetric and asymmetric broadening of the $\mathrm{j}^{\text {th }}$ process. $\Delta \varepsilon_{\mathrm{j}}$ is its dielectric strength, and $\tau_{\mathrm{HN}}$ its characteristic relaxation time. It was not possible to reproduce the broad maximum in Fig. 1 with only a single $\mathrm{HN}$-function, a tentative description being shown in the ESI.

Interfacial processes are described using a Debye function (HN function with $\gamma=\delta=1)^{16}$. However, MWS-processes observed in nanocomposites are commonly described by a 
symmetric Cole-Cole function $(\gamma \neq 1, \delta=1)^{11,18,19}$, the broadening of which can be seen as a result of superposition of Debye processes. This is the case for our MWS2 process, whereas an asymmetric distribution of times was necessary to fit the data of MWS1 $(\gamma, \delta \neq 1)$. Note that the fitting procedure using eq 1 was carried out simultaneously on $\varepsilon$ ' and $\varepsilon^{\prime \prime}$, and that the number of fit parameters was reduced by a two-step procedure, where the almost Tindependent shape parameters have been fixed to their average value: $\gamma_{\mathrm{MWS} 2}=0.55, \gamma_{\mathrm{MWS} 1}=$ 0.42 , and $\delta_{\mathrm{MWS} 1}=0.53$. In order to improve the fitting quality in some rare cases, $\gamma_{\mathrm{MwS} 1}$ was allowed to fluctuate within 5\% (see ESI for details). The dielectric strengths were also found to vary hardly with T, MWS1 being significantly more intense than MWS2 $\left(\Delta \varepsilon_{\mathrm{MWS} 1}=1.37 \pm\right.$ $\left.0.07, \Delta \varepsilon_{\mathrm{MWS} 2}=0.43 \pm 0.05\right)$ at $8.4 \%$ vol. To summarize, these slow, high-temperature processes are shown to be associated with a broad distribution of relaxation times described by $\gamma$-values considerably smaller than one. As recalled in the introduction, we are dealing with a highly polydisperse system ${ }^{6}$, in which a broad distribution of aggregate sizes introduces a variety of characteristic lengths either of aggregates or distances between them.

The two MWS-processes described in Fig. 1 do not exist in absence of silica. Indeed, any pure matrix contribution is at least two orders of magnitude weaker. To make this clear, all data reported here are compared explicitly to the matrix contribution in the ESI. It is therefore straightforward to check the influence of the silica volume fraction. The dielectric response for samples with $\Phi_{\mathrm{si}}=8.4-21.1 \% \mathrm{vol}$ is shown in Fig. 2 for $\mathrm{T}=333 \mathrm{~K}$. As observed in the $8.4 \%$ vol-nanocomposite, the two MWS-processes enter the frequency window above $253 \mathrm{~K}$, and are well visible at $333 \mathrm{~K}$. 

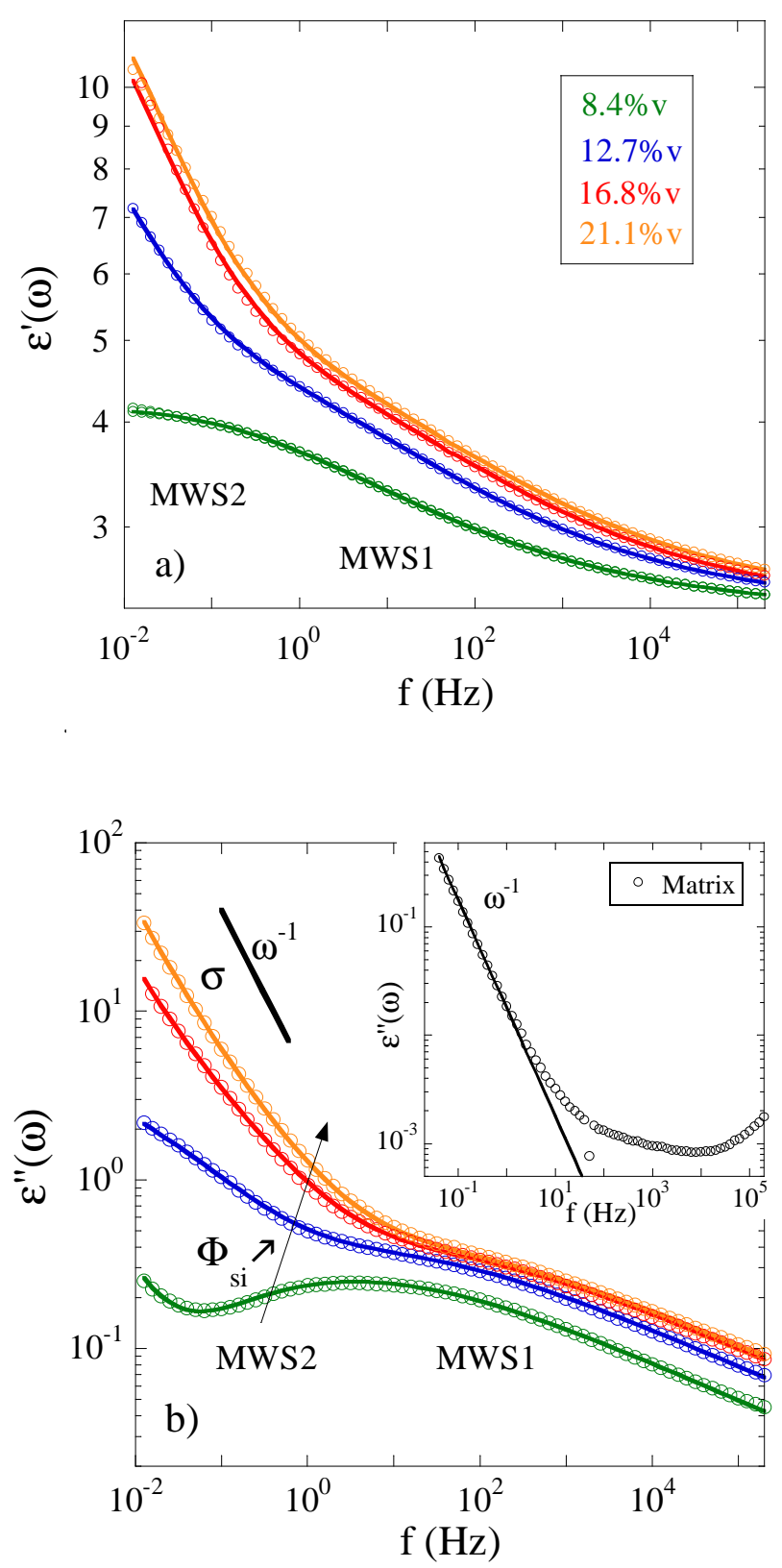

Fig. 2 Dielectric spectra at $333 \mathrm{~K}$ for nanocomposites with different silica volume fractions $\left(\Phi_{\mathrm{si}}=8.4\right.$, 12.7, 16.8 and $21.1 \%$ vol from bottom to top, $50 \%$ D3). a) Real and b) imaginary permittivity data. Solid lines are fits to the experimental data by means of eq 1 . Individual contributions to the dielectric loss are shown in the ESI. Inset: same data for the pure matrix.

In Fig. 2a and b, an increase of the dielectric signal with the filler fraction is observed for both $\varepsilon^{\prime}(\omega)$ and $\varepsilon^{\prime \prime}(\omega)$, respectively. For comparison, the dielectric loss of the pure matrix is shown in the inset of Fig. $2 b$ (see also ESI). The strong rise of the real part at low frequencies (Fig. 2a) indicates that such an increase of the permittivity is linked to a relaxation process and not only to the strong contribution proportional to $\omega^{-1}$ of the dc-conductivity observed in Fig. 2 b. Indeed, as with the $8.4 \%$ vol-sample, the dielectric spectra in this temperature range cannot be 
fitted with only one HN contribution (eq 1), in addition to the dc-conductivity (see ESI). Note that, for all filler contents, we used the same approach of temperature independent shape parameters $\left(\gamma_{\mathrm{i}}\right.$ and $\left.\delta_{\mathrm{i}}\right)$ for both MWS-processes. These parameters were not found to evolve with $\Phi_{\mathrm{si}}$, in agreement with the assumption of a constant polydispersity in ref ${ }^{6}$. For a better visualization of the two processes (MWS1, MWS2), the modulus representation, which reduces the effect of dc-conductivity, is shown in the ESI.

The influence on the dielectric data of drying has been evidenced in Figure 3 for a nanocomposite at $8.4 \%$ v. Samples have undergone the procedure outlined in the Materials section, which lead to water losses of the order of about $1 \%$ w. Clearly, the relaxation time associated with the MWS1 process is affected by drying, as it moves to lower frequencies by more than a decade, in agreement with ${ }^{11}$. On the other hand, the characteristic time of the MWS2 process does not seem to be affected by drying. The dielectric strength of MWS2 increases by some $30 \%$, which is small with respect to the absolute variation of the strengths with silica volume fraction as discussed later in the article. Note also that the ionic conductivity is identical before and after drying. To summarize, neither MWS2 nor conductivity are sensitive to the water content.

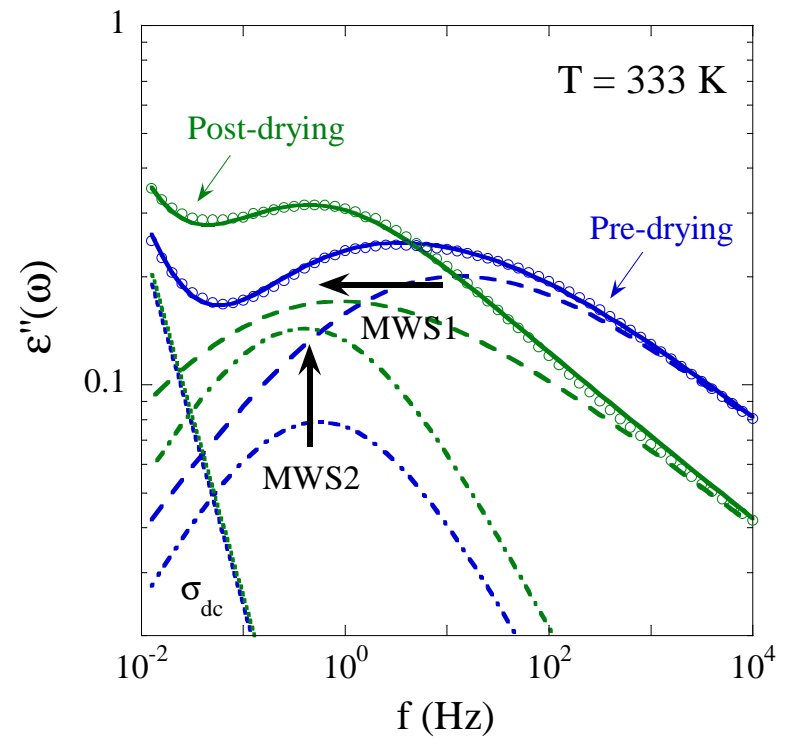

Fig. 3 Imaginary permittivity data at $333 \mathrm{~K}$ for a $\left(\Phi_{\mathrm{si}}=8.4 \% \mathrm{v}, 50 \% \mathrm{D} 3\right)$-nanocomposite before and after drying. Solid lines are fits to the experimental data by means of eq 1 . The relaxation time of the individual contribution MWS1 (dashed lines) is seen to be sensitive to drying, whereas MWS2 (dashed-dotted lines) remains at the same position. Pre- and post-drying ionic conductivity are shown as superimposed dotted lines. 
The study of the temperature dependence of the relaxation times of the high-temperature interfacial processes in presence of filler gives information about possible common origins of processes. For both dielectric processes, the relaxation time corresponding to the maximum of the dielectric loss, $\tau_{\max }=\left(2 \pi f_{\max }\right)^{-1}$, is a convenient model-free parameter. It has been determined from the fit parameters as a function of temperature. Their evolution with $\mathrm{T}$ follows an Arrhenius law describing thermally activated processes

$$
\tau(T)=\tau_{0} \exp \left(\frac{E}{k_{B} T}\right)
$$

where $\mathrm{E}$ is the activation energy, $\mathrm{k}_{\mathrm{B}}$ the Boltzmann constant and $\tau_{0}$ the high-temperature limit associated with vibrational times. A similar temperature dependence is thus expressed by a close activation energy. In this article, we concentrate on MWS2. Its relaxation map, i.e. its temperature dependence, is shown in Fig. 4 together with the one of the dc-conductivity for nanocomposites of different filler volume fractions. For the sake of comparison, the negative logarithm of $\sigma_{\mathrm{dc}}$ is plotted.

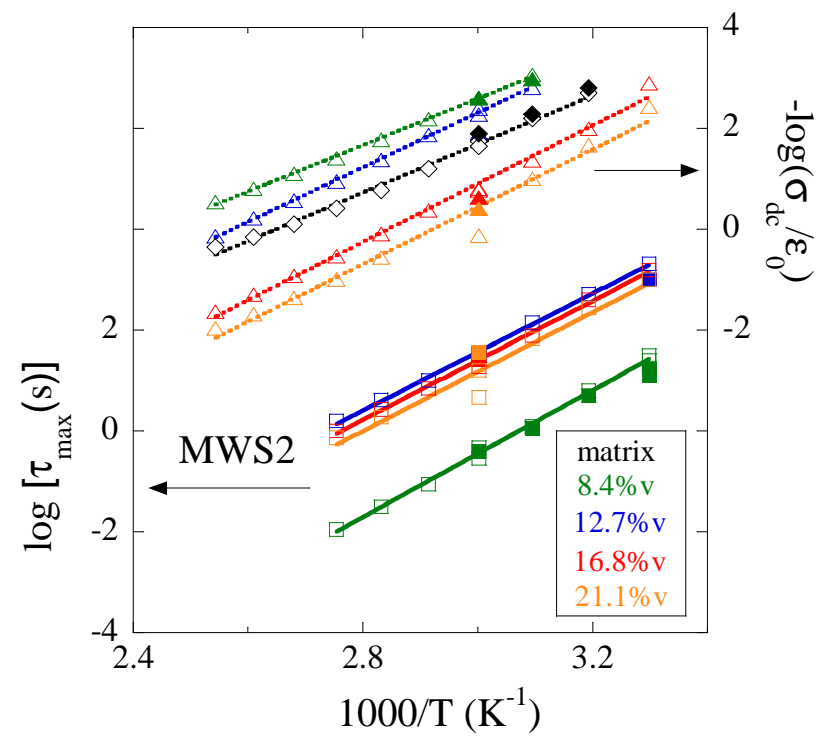

Fig. 4 Dynamics of MWS2-process as a function of the reciprocal temperature for different filler fractions $(8.4,12.7,16.8$ and $21.1 \%$ vol) at $50 \% \mathrm{D} 3$ (empty squares). Solid lines are fits by means of the Arrhenius equation. The temperature dependence of the logarithm of dc-conductivity (empty triangles) is included with linear fits (dotted lines). Black empty diamonds refer to the conductivity of the matrix. Plain symbols for MWS2 and conductivity of all samples after drying are also shown at selected temperatures. 
In Fig. 4, the conductivity increases with the amount of silica filler as shown explicitly in Fig. 2, and discussed later. Both for conductivity and MW2, the influence of drying is shown to be negligible as already seen in Fig. 3 for one temperature. The corresponding empty symbols are found to superimpose with the data of the samples before drying. The temperature dependence of the inverse of the conductivity parallel to the one of the MWS2 process suggests a close relationship with this latter process. Indeed, an Arrhenius fit of MWS2 leads to an average activation energy of $\mathrm{E}_{\mathrm{MWS} 2}=114 \mathrm{~kJ} / \mathrm{mol}$, without tendency with $\Phi_{\mathrm{si}}$, and similar values are found for the conductivity, as summarized in Table 1.

Table 1: Activation energies for the MWS2-process and the dc-conductivity in the pure matrix and in nanocomposites, both with $50 \% \mathrm{D} 3$.

\begin{tabular}{|r|r|r|}
\hline \multicolumn{1}{|c|}{$\boldsymbol{\Phi}_{\text {si }}(\boldsymbol{\%}$ vol $)$} & $\mathbf{E}_{\text {MWS } 2}(\mathbf{k J} / \mathbf{m o l})$ & \multicolumn{1}{|c|}{$\mathbf{E}_{\boldsymbol{\sigma}}(\mathbf{k J} / \mathbf{m o l})$} \\
\hline 0 & - & 92 \\
\hline 8.4 & 120 & 88 \\
\hline 12.7 & 111 & 103 \\
\hline 16.8 & 113 & 109 \\
\hline 21.1 & 113 & 110 \\
\hline
\end{tabular}

The $\alpha$-process has been characterized following the same procedure (eq 1) and an apparent activation energy of $80 \mathrm{~kJ} / \mathrm{mol}$ has been found in the high-temperature range, between 283 and $313 \mathrm{~K}$. Note that neither the spectral shape nor the relaxation time were affected by the addition of silica. On the other hand, $92 \mathrm{~kJ} / \mathrm{mol}$ was found for the activation energy of the dcconductivity in the pure matrix. The close agreement between these two values indicates that the latter is primarily controlled by matrix properties, as well as of course by the presence of

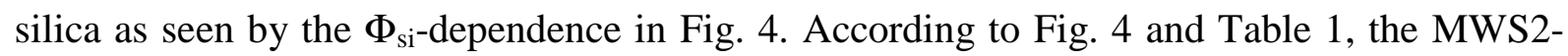
process has activation energies comparable to the conductivity, and independent of the silica fraction. This similarity suggests that the dynamics of MWS2 is also controlled by the polymer matrix. As can be seen in Fig. 4, both the conductivity and the MWS2 time scales depend on the silica fraction. It follows that the dynamics of MWS2 relates also to the nanocomposite structure. There are thus two different conduction mechanisms, one through the matrix between aggregates, and another one along the surface of aggregates. Note that we have detailed information on aggregate structure within branches, see ref ${ }^{6}$. From the preceding arguments, it follows that aggregates are not in close contact in one branch, but 
must be surrounded by a polymer layer controlling the transfer of charges by a hopping mechanism from aggregate to aggregate surface. Finally, concerning the length scales, the MWS1 process in silica nanocomposites has been attributed to the hydration layer on the surface of the NPs ${ }^{11}$, which defines the length scale of the process. Taking into account that the MWS2 time-scale is much longer than for MWS1, it is concluded that the relevant processes must take place at larger length scales, i.e. ensembles of aggregates, up to percolation.

As a last point about Fig. 4, the relaxation times $\tau_{\mathrm{MWS} 2}$ lie in the same range from 12.7 to $21.1 \%$ vol, but they are notably slower ( $\approx 60$ times) than in the $8.4 \%$ vol-nanocomposite. Such a slow-down with increasing $\Phi_{\text {si }}$ cannot be caused by a smaller diffusion rate as the conductivity increases with $\Phi_{\mathrm{si}}$. It should rather be related to larger distances involved in the transfer of charges, and linked to the formation of a percolated network of aggregates, with polymeric paths in between. This explanation agrees with the evolution of the dielectric strength of MWS2 with $\Phi_{\mathrm{si}}$, which is shown in Fig. 5 to have a significant jump around the mechanical percolation threshold of $12 \% \mathrm{vol}^{6}$. As we know from our detailed structural study, percolation is accompanied by structural rearrangements. In particular, increasing the silica fraction leads to denser fractal branches ${ }^{6}$. In our structural model, this density was expressed by the volume fraction of aggregates, which was found to increase from $47 \%$ vol to $69 \%$ vol, for $8.4 \%$ vol to $21 \%$ vol silica, respectively. Note that such large values can only be reached with strongly polydisperse aggregates. The polymer matrix influences thus less and less the conduction mechanism governed by conducting paths along the silica aggregates as the silica content increases and the branches become more densely filled with polydisperse aggregates. The temperature dependence of the dielectric strengths obtained for both MWS-processes is given in Fig. 5 for the different silica volume fractions. Within error bars, $\Delta \varepsilon_{\mathrm{MWSi}}$ do not evolve with temperature. The dielectric strength of MWS1 increases slightly with $\Phi_{\text {si }}$ whereas the dielectric strength of MWS2 is seen to increase strongly. In both cases, it seems to saturate at the highest silica fractions. Coming back to the discussion of the strengths, the scattering of the data points for a given volume fraction in Fig. 5 is compatible with the discrepancy in Fig. 3. The corresponding data point (dried state) has been added for clarity in Fig 5 . 


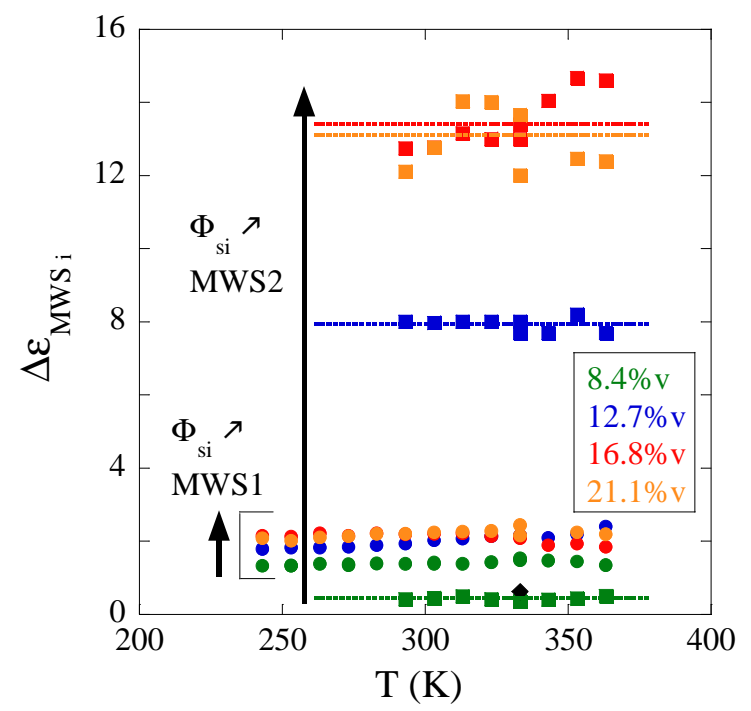

Fig. 5 Dielectric strength of MWS1- (circles) and MWS2-processes (squares) as a function of temperature for nanocomposites with different filler fractions and 50\%D3. Dotted lines are the average MWS2-strengths over T. The dielectric strength of MWS2 after drying is shown at $60^{\circ} \mathrm{C}$ (Fig. 3, $8.4 \% \mathrm{v}$ silica) as a black diamond.

The average values of $\Delta \varepsilon_{\mathrm{MWSi}}$ as a function of the filler fraction are reported in Fig. 6, together with the dc-conductivity obtained at the highest temperature (393 K), i.e., where it takes its maximal value.

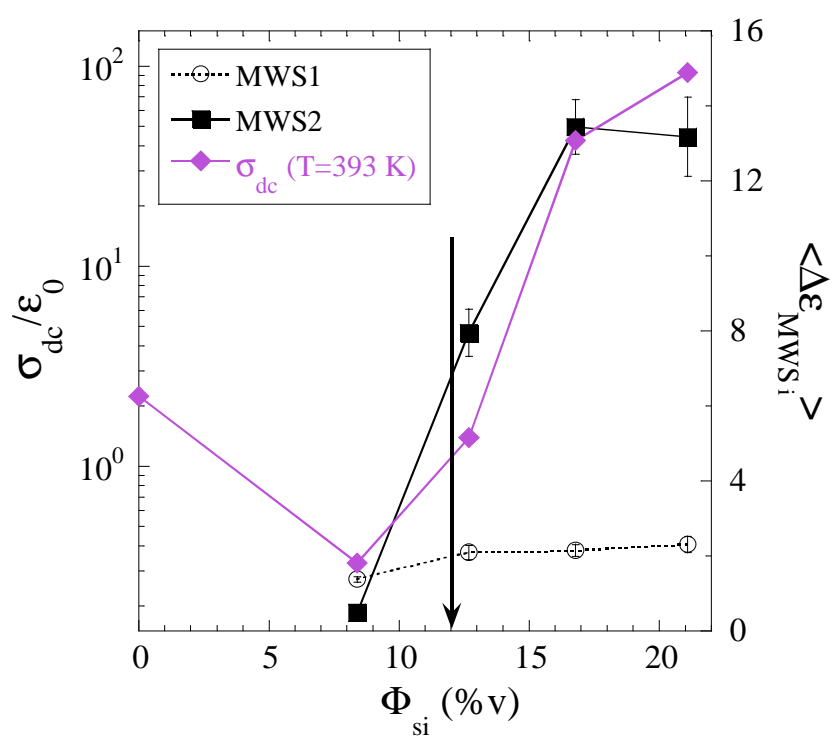

Fig. 6 DC-conductivity at $393 \mathrm{~K}$ (diamonds) and average value of the dielectric strengths over $\mathrm{T}$ for MWS1 (circles) and MWS2 (squares) as a function of filler fraction $(50 \% \mathrm{D} 3)$. Lines are guide for the eyes. The percolation threshold as obtained from rheology ${ }^{6}$ is indicated by an arrow. 
Fig. 6 shows that the MWS1 process is rather insensitive to the silica content, whereas the ionic conductivity displays an evolution with $\Phi_{\text {si }}$ strong enough to justify a log scale. Although somewhat weaker and plotted on linear scale, the strength of MWS2 also increases considerably for $\Phi_{\mathrm{si}} \geq 8.4 \% \mathrm{vol}$, in analogy with the logarithm of the dc-conductivity. Between 0 and $8.4 \%$ vol, $\sigma_{\mathrm{dc}}$ first decreases with $\Phi_{\mathrm{si}}$. We do not have a quantitative explanation for this experimental observation. However, it is compatible with the trapping of free charge carriers initially present in neat SB at the silica surface. Above $8.4 \%$ vol, however, the contribution of free charge carriers cannot be invoked to explain the strong increase of $\sigma_{\mathrm{dc}}$. Indeed, the surface electrical conductivity of silica is linked to the concentration of adsorbed water and silanol groups, which affect the number of charge carriers and the dielectric constant. ${ }^{20}$ Concerning water, the conductivity before and after drying is identical (Fig 4), which suggests that water does not participate in conduction. On the other hand, hydroxyl groups may dissociate to form free protons, which can migrate along the silica surface ${ }^{20}$. Here, the number of $\mathrm{Si}-\mathrm{OH}$ increases proportionally to the silica fraction if we neglect minor modifications due to the decrease of the grafting density with $\Phi_{\mathrm{si}}$ at constant percentage of graftable chains, $50 \% \mathrm{D} 3$. Note that the grafting density is smaller than $0.2 \mathrm{~nm}^{-2}$, as compared to 4 to 5 available silanols per $\mathrm{nm}^{2}{ }^{21}$. It follows that the strong conductivity increase (by more than two orders of magnitude) observed between 8.4 to $16.8 \%$ vol in Fig. 6 cannot be explained solely by an increase of the charge carrier concentration, and suggests the formation of large clusters or of a percolated silica network through the sample. This agrees with the mechanical percolation threshold estimated to $12 \%$ vol silica by means of rheology on the same samples ${ }^{6}$. To summarize, both conductivity and the new MWS2-process are thus tools, which are sensitive to large-scale structural reorganization. We have shown that the strength of MSW2 is mostly unaffected by drying, but increases strongly as the silica content is increased above the percolation threshold. Moreover, the activation energy of MWS2 gives additional information on the existence of polymer layers between aggregates.

\section{CONCLUSION}

We have provided evidence for two different interfacial dielectric processes. One of them may be related to hydration layers on silica NPs ${ }^{11}$, i.e. to a rather local phenomenon on the scale of a few NPs. Its strength is comparatively independent of the silica content, and percolation. Comparison before and after drying proved that the small quantities of water 
affect the MWS1 process, but not conductivity and new high-temperature dielectric process MWS2. The latter are on the contrary dominated by nanocomposite microstructure, and in particular percolation of the aggregates of silica NPs within space-filling branches. More specifically, the strength of MWS2 follows the percolation behavior observed both in rheology and in the ionic conductivity, whereas the MWS2 activation energy hints at a control of the dynamics by diffusion processes through the matrix between filler aggregates. In the framework of comprehensive multi-scale dynamical studies, it is hoped that the identification and description of the MWS2-process provide a straightforward tool to interpret data of complex nanocomposite samples.

Acknowledgements: We are thankful for a "Chercheur d'Avenir" grant by the LanguedocRoussillon region (J.O.) and Ph.D. funding "CIFRE" by Michelin (G.P.B.). The authors acknowledge financial support from the European Commission under the Seventh Framework Program by means of the grant agreement for the Integrated Infrastructure Initiative N. 262348 European Soft Matter Infrastructure (ESMI).

Electronic Supplementary Information (ESI) available: Complementary dielectric data for the SB-silica nanocomposites, and details about the fit contributions. See DOI: 10.1039/b000000x/

\section{References}

1. J. Jancar, J. F. Douglas, F. W. Starr, S. K. Kumar, P. Cassagnau, A. J. Lesser, S. S. Sternstein and M. J. Buehler, Polymer, 2010, 51, 3321-3343.

2. G. Heinrich, M. Kluppel and T. A. Vilgis, Current Opinion in Solid State \& Materials Science, 2002, 6, 195-203.

3. G. Schmidt and M. M. Malwitz, Current Opinion in Colloid \& Interface Science, 2003, 8, 103-108.

4. J. E. Mark, B. Erman and F. R. Eirich, Science and Technology of Rubber, Academic Press, San Diego, 1994.

5. M. Mokhtari, D. Schipper and T. Tolpekina, Tribol Lett, 2014, 54, 297-308. 
6. G. P. Baeza, A. C. Genix, C. Degrandcourt, L. Petitjean, J. Gummel, M. Couty and J. Oberdisse, Macromolecules, 2013, 46, 317-329.

7. G. P. Baeza, A. C. Genix, C. Degrandcourt, L. Petitjean, J. Gummel, R. Schweins, M. Couty and J. Oberdisse, Macromolecules, 2013, 46, 6388-6394.

8. G. P. Baeza, A. C. Genix, C. Degrandcourt, J. Gummel, M. Couty and J. Oberdisse, Soft Matter, 2014, 10, 6686-6695.

9. G. P. Baeza, A. C. Genix, C. Degrandcourt, J. Gummel, A. Mujtaba, K. Saalwachter, T. Thurn-Albrecht, M. Couty and J. Oberdisse, ACS Macro Letters, 2014, 3, 448-452.

10. M. Bohning, H. Goering, A. Fritz, K. W. Brzezinka, G. Turky, A. Schonhals and B. Schartel, Macromolecules, 2005, 38, 2764-2774.

11. J. Otegui, G. A. Schwartz, S. Cerveny, J. Colmenero, J. Loichen and S. Westermann, Macromolecules, 2013, 46, 2407-2416.

12. J. Mijovic, H. K. Lee, J. Kenny and J. Mays, Macromolecules, 2006, 39, 2172-2182.

13. J. G. Meier and M. Kluppel, Macromolecular Materials and Engineering, 2008, 293, 12-38.

14. J. E. Mark, Physical properties of polymers handbook, AIP Press, Woodbury New York, 1996.

15. G. Ghosh, Opt. Commun., 1999, 163, 95-102.

16. F. Kremer and A. Schönhals, Broadband Dielectric Spectroscopy, Springer-Verlag, Heidelberg, 2003.

17. S. Havriliak and S. Negami, Journal of Polymer Science Part C-Polymer Symposium, 1966, 14, 99-\&.

18. Y.-H. Lee, A. J. Bur, S. C. Roth and P. R. Start, Macromolecules, 2005, 38, 3828-3837.

19. J. K. Jeszka, L. Pietrzak, M. Pluta and G. Boiteux, Journal of Non-Crystalline Solids, 2010, 356, 818-821.

20. J. H. Anderson and G. A. Parks, J. Phys. Chem., 1968, 72, 3662-\&.

21. R. K. Iler, The Chemistry of Silica, John Wiley and Sons, New York, 1979. 